\title{
KÄHLER MOIŠEZON SPACES WHICH ARE PROJECTIVE ALGEBRAIC
}

\author{
CHARLES VUONO
}

(Communicated by Peter Li)

\begin{abstract}
The Moišezon theorem is extended to complex spaces with isolated singularities.
\end{abstract}

A problem of fundamental concern is determining sufficient conditions under which a complex space $X$ is algebraic. Ideally, these conditions involve simple intrinsic analytic features of $X$. An $n$-dimensional manifold $X$ is called Kähler if it admits a hermitian metric whose associated $(1,1)$-form $\omega$ is closed, and it is called Moišezon if it admits $n$ algebraically independent global meromorphic functions. A fundamental theorem of Moišezon states that if a manifold $X$ is Kähler and Moišezon, then $X$ is a projective algebraic variety (i.e., $X$ is the common zero locus of a set of homogeneous polynomials in projective space). The Kähler and Moišezon conditions can be generalized to singular complex spaces; however, it is no longer the case that these two conditions will imply algebraicity (an example is provided in [7]). In this paper we investigate situations under which a complex space $X$ which is Kähler and Moišezon must be projective algebraic. For example, we show that if a compact space of dimension at least four is a complete intersection with isolated singularities, then the Kähler and Moišezon conditions imply projective algebraicity.

In Section 1 we investigate conditions which allow us to extend holomorphic line bundles over singular points of a complex space. In Section 2 we use a singular version of the Kodaira embedding theorem to reduce the problem to one of extending line bundles over singular points.

\section{EXTENDING LINE BUNDLES OVER SINGULAR POINTS}

Let $X$ be a complex space with isolated singularities and structure sheaf $\mathscr{O}_{X}$. Recall that an invertible sheaf is a locally free $\mathscr{O}_{X}$-module of rank one and that the set of these is the Picard group $H^{1}\left(X, \mathscr{O}_{X}^{*}\right)$. We seek sufficient conditions for a holomorphic line bundle $L$ on the regular part of $X$ to extend to $X$ as an invertible sheaf. For a small neighborhood $U$ of a singularity $x \in X$, the exact sequence of sheaves $0 \rightarrow \mathbb{Z} \rightarrow \mathscr{O}_{X} \rightarrow \mathscr{O}_{X}^{*} \rightarrow 0$ and the exact sequence

Received by the editors June 7, 1993.

1991 Mathematics Subject Classification. Primary 32J25.

Key words and phrases. Kähler space, Moišezon space, projective embedding. 
for cohomology with supports induce the following commutative diagram with exact rows and exact columns:

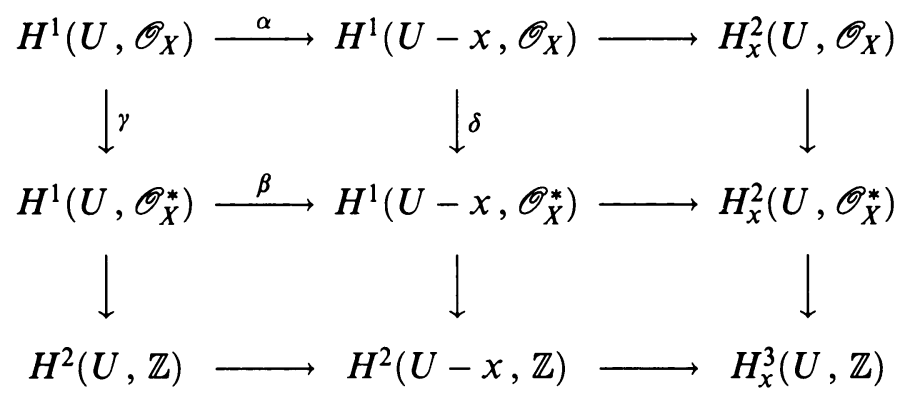

If $\beta$ is surjective, then any line bundle $L$ over $U-x$ must extend to $U$. Among the several possibilities, the most useful way to establish the surjectivity of $\beta$ is to suppose that $H_{x}^{2}\left(U, \mathscr{O}_{X}\right)=H^{2}(U-x, \mathbb{Z})=0$. In this case, $\alpha$ and $\delta$ are surjective, and the representative of $L$ in $H^{1}\left(U-x, \mathscr{O}_{X}^{*}\right)$ lifts to $H^{1}\left(U, \mathscr{O}_{X}\right)$ and maps down to $H^{1}\left(U, \mathscr{O}_{X}^{*}\right)$ via $\gamma$. By the commutativity of the diagram, this gives a line bundle over $U$ whose representative in $H^{1}\left(U, \mathscr{O}_{X}^{*}\right)$ restricts to the representative of $L$ in $H^{1}\left(X, \mathscr{O}_{X}^{*}\right)$. We have established the following lemma:

Lemma 1.1. If $X$ is a complex space with isolated singularities for which there exists a sufficiently small neighborhood $U$ of each singularity $x$ with $H_{x}^{2}\left(U, \mathscr{O}_{X}\right)$ $=H^{2}(U-x, \mathbb{Z})=0$, then any holomorphic line bundle $L$ over the regular part of $X$ is the restriction of an invertible sheaf on $X$.

The cohomological conditions of Lemma 1.1 are known to hold under more natural conditions on $X$. After recalling some pertinent definitions, we shall recall results of this type.

Definition 1.2. The homological codimension of a coherent analytic sheaf $\mathscr{F}$ (denoted $\operatorname{codh}_{x} \mathscr{F}$ ) over an $n$-dimensional complex space $X$ at a point $x$ is $n-k$ where $k$ is defined as the smallest integer such that there exists a resolution of $\mathscr{F}$

$$
0 \rightarrow P_{k} \stackrel{d_{k}}{\longrightarrow} P_{k-1} \stackrel{d_{k-1}}{\longrightarrow} \cdots \rightarrow P_{0} \rightarrow \mathscr{F}_{x} \rightarrow 0
$$

where each $P_{i}$ is projective. The homological codimension of a sheaf is defined by $\operatorname{codh} \mathscr{F}:=\inf _{x \in X} \operatorname{codh}_{x} \mathscr{F}$, and the homological codimension of $X$, $\operatorname{codh} X$, is the homological codimension of the structure sheaf $\mathscr{O}_{X}$.

High homological codimension is useful in establishing vanishing of cohomology with supports. In particular, the following theorem shows that $\operatorname{codh} X \geq 3$ implies the vanishing of $H_{x}^{2}\left(U, \mathscr{O}_{X}\right)$ as required in Lemma 1.1.

Theorem 1.3 [9, Theorem 1.14]. Let $\left(X, \mathscr{O}_{X}\right)$ be a complex space and $x \in X$. Then for $U$ a small neighborhood of $x, H_{x}^{i}\left(U, \mathscr{O}_{X}\right)=0$ for $i \leq q$ iff $\operatorname{codh} X \geq$ $q+1$.

Results of Hamm provide natural conditions under which $H^{2}(U-x, \mathbb{Z})$ vanishes. Recall that $U$ is biholomorphic to the common zero locus of $s$ analytic equations in $\mathbb{C}^{N}$. Let $N(x)$, the embedding dimension at $x$, be the least possible $N$, and accordingly let $r(x):=N(x)-s(x)$. 
Theorem 1.4 [3, 4]. Let $X$ be an n-dimensional irreducible complex space with isolated singularities. For a small enough neighborhood $U$ of $x$, $H^{i}(U-x, \mathbb{Z})=0$ if either $0<i \leq r(x)-2$ or $0<i \leq 2 n-N(x)-1$.

Summarizing we have

Theorem 1.5. Suppose $X$ is an n-dimensional complex space with isolated singularities such that $\operatorname{codh} X \geq 3$ and either $r(x) \geq 4$ or $2 n-N(x) \geq 3$ at all singular points $x$. If $L$ is a holomorphic line bundle on the regular part of $X$, then $L$ is the restriction of an invertible sheaf on $X$.

Recall that a complex space is called Cohen-Macaulay if $\operatorname{codh}_{x} \mathscr{O}_{X}=\operatorname{dim}_{x} X$ at all points $x \in X$.

Corollary 1.6. Suppose $X$ is a complex space of dimension $n \geq 4$ with isolated singularities which is Cohen-Macaulay, and suppose that either $r(x) \geq 4$ or $2 n-N(x) \geq 3$ for all singular points $x$. Then any holomorphic line bundle on the regular part of $X$ is the restriction of an invertible sheaf on $X$.

A complex space $X$ of dimension $n$ is called a local complete intersection if $r(x)=n$ for all $x \in X$.

Corollary 1.7 [2, Theorem 3.13.ii]. If a complex space $X$ is a local complete intersection of dimension at least four, then any holomorphic line bundle $L$ on the regular part of $X$ is the restriction of an invertible sheaf on $X$.

Proof. By [1, p. 200], local complete intersections are Cohen-Macaulay.

\section{Projective EMBeddings OF SPACES WITH ISOLATED SINGULARITIES}

A function $f$ on a singular space $X$ is meromorphic (respectively plurisubharmonic, pluriharmonic) if at all points $x \in X$ there exists a neighborhood $U$ of $x$ embedded in $\mathbb{C}^{N}$ such that $f$ is the restriction of a meromorphic (respectively plurisubharmonic, pluriharmonic) function on $\mathbb{C}^{N}$. A compact irreducible complex space $X$ is called Moišezon if the transcendence degree of the field of meromorphic functions on $X$ equals the complex dimension of $X$. A Kähler space is a complex space $X$ such that there exists an open covering $\mathscr{U}_{A}$ of $X$ and strictly plurisubharmonic smooth functions $\phi_{U}$ for each $U \in \mathscr{U}$ such that $\phi_{U}-\phi_{V}$ is pluriharmonic on $U \cap V$ for all $U, V \in \mathscr{U}$. These definitions generalize the standard definitions of Moišezon and Kähler manifolds; in particular, the forms $\sqrt{-1} \partial \bar{\partial} \phi_{U}$ piece together to be a Kähler metric on the regular part of $X$.

A previous result of the author provides a Kodaira-type embedding theorem for Kähler spaces with isolated singularities:

Theorem 2.1 [10]. Let $X$ be a compact normal Kähler space with isolated singularities. Suppose $X$ admits an invertible sheaf $\mathscr{L}$ whose restriction to the regular part of $X$ is a positive holomorphic line bundle $L$. Then $X$ is a projective algebraic variety.

Remark 2.2. Without the Kähler hypothesis, a theorem of Riemenschneider [8] shows that $X$ is Moišezon. 
The usefulness of this theorem is that no notion of positivity of a line bundle $L$ is required near a singular point $x$ of $X$. All that is necessary is that $L$ extends over $x$ and defines rational maps $\sigma_{L^{p}}: X \rightarrow \mathbb{P}^{N}$ given by global holomorphic sections of $L^{p}$.

We now construct a positive line bundle on the regular part of a complex space which is both Kähler and Moišezon.

Theorem 2.3 [6]. Every compact irreducible Moišezon space $X$ has a projective algebraic desingularization $\pi_{i}: \tilde{X} \rightarrow X$. In fact, there exists a finite sequence of monoidal transformations $\phi: X_{i+1} \rightarrow X_{i}, 0 \leq i<r$, such that

(i) $X_{0}=X$;

(ii) the center $Z_{i}$ of $\phi_{i}$ is a nonsingular projective algebraic variety which is nowhere dense in $X_{i}$;

(iii) $Z_{i} \subseteq\left\{\right.$ the singular set of $\left.X_{i}\right\}$ if $X_{i}$ is singular;

(iv) $X_{r}$ is a projective algebraic manifold.

Theorem 2.4. Suppose $X$ is a normal irreducible complex space with isolated singularities such that any holomorphic line bundle on the regular part of $X$ is the restriction of an invertible sheaf on $X$. If $X$ is Kähler and Moišezon, then $X$ is projective algebraic.

Proof. By Theorem 2.3, there exists a desingularization $\pi: \tilde{X} \rightarrow X$ with exceptional set $E$ and $\pi(E)$ supported on the singular set of $X$. Since $\tilde{X}$ is both Kähler and Moišezon, $\pi$ is in fact a projective algebraic desingularization. Therefore, the positive hyperplane bundle $\mathscr{O}(1)$ over the Zariski open set $\tilde{X}-E$ of the projective algebraic manifold $\tilde{X}$ pushes forward to be a positive line bundle on the regular part of $X$. The result follows from Theorem 2.1.

We combine Theorem 2.4 with the results of Section 1 to assert our main results.

Theorem 2.5. Let $X$ be a normal irreducible $n$-dimensional complex space with isolated singularities such that $\operatorname{codh} X \geq 3$ and either $r(x) \geq 4$ or $2 n-N(x)$ $\geq 3$ at all singular points $x \in X$. If $X$ is Kähler and Moišezon, then $X$ is a projective variety.

Proof. The theorem follows from Theorem 1.5 and Theorem 2.4.

Remark 2.6. When $\operatorname{codh} X \geq 4$, the normality assumption found in Theorem 2.5 is automatically satisfied [5].

Corollary 2.7. Let $X$ be an irreducible Cohen-Macaulay complex space with isolated singularities and of dimension at least four. Suppose that either $r(x) \geq 4$ or $2 n-N(x) \geq 3$ for all singular points $x \in X$. If $X$ is Kähler and Moišezon, then $X$ is a projective algebraic variety.

Corollary 2.8. Suppose $X$ is an irreducible local complete intersection of dimension at least four and with isolated singularities. If $X$ is Kähler and Moišezon, then $X$ is a projective algebraic variety.

\section{ACKNOWLEDGMENT}

We thank János Kollár for pointing out that Corollary 1.7 is given by [2, Theorem 3.13.ii]. 


\section{REFERENCES}

1. A. Andreotti and H. Grauert, Théoremes de finitude pour la cohomologie des espaces complexes, Bull. Soc. Math. France 90 (1962), 193-259.

2. A. Grothendieck, Cohomologie locale des faisceaux coherents et theoremes de Lefschetz locaux et globaux, Adv. Stud. Pure Math., vol. 2, North-Holland, Amsterdam, 1962.

3. H. A. Hamm, Lokale topologische Eigenschaften komplexer Räume, Math. Z. 19 (1971), 235-252.

4. - On the vanishing of local homotopy groups for isolated singularities of complex spaces, J. Reine Angew. Math. 323 (1981), 172-176.

5. A. Markoe, $A$ characterization of normal analytic spaces by the homological codimension of the structure sheaf, Pacific J. Math. 52 (1974), 485-489.

6. B. G. Moišezon, Resolution theorems for compact complex spaces with a sufficiently large field of meromorphic functions, Izv. Akad. Nauk SSSR Ser. Mat. 31 (1967), 1331-1356; English transl., Math. USSR-Izv. 1 (1967), no. 6, 1331-1356.

7. —_, Singular Kählerian spaces, Manifolds-Tokyo 1973 (A. Hattori, ed.), Univ. of Tokyo Press, Tokyo, 1975, pp. 343-351.

8. O. Riemenschneider, Characterizing Moišezon spaces by almost positive coherent analytic sheaves, Math. Z. 123 (1971), 263-284.

9. Y. T. Siu and G. Trautmann, Gap-sheaves and extension of coherent analytic subsheaves, Lecture Notes in Math., vol. 172, Springer-Verlag, New York, 1971.

10. C. Vuono, The Kodaira embedding theorem for Kähler varieties with isolated singularities, J. Geom. Anal. 3 (1993).

Department of Mathematics, University of Utah, Salt Lake City, Utah 84112

Current address: Department of Mathematics, School of Science, Nagoya University, Chikusaku, Nagoya 464-01, Japan

E-mail address: vuono@math.nagoya-u.ac.jp 